\title{
Puerarin suppresses production of nitric oxide and inducible nitric oxide synthase in lipopolysaccharide- induced N9 microglial cells through regulating MAPK phosphorylation, O-GIcNAcylation and NF- $\kappa B$ translocation
}

\author{
GAO-MING ZHENG ${ }^{1 *}$, CHAO YU ${ }^{2 *}$ and ZHU YANG ${ }^{1}$ \\ ${ }^{1}$ Department of Obstetrics and Gynecology, the Second Affiliated Hospital of Chongqing Medical University, \\ Chongqing 400010; ${ }^{2}$ Institute of Life Sciences, Chongqing Medical University, Chongqing 400016, P.R. China
}

Received November 4, 2011; Accepted December 22, 2011

DOI: 10.3892/ijo.2012.1331

\begin{abstract}
Microglial cells play a critical role in mediating central nervous system inflammatory processes. Activated microglial cells induced by proinflammatory factor, such as lipopolysaccharide (LPS), release many kinds of neurotoxic cytokines including reactive oxygen species (ROS) which contributes to the pathogenesis of neurodegenerative diseases. Puerarin, extracted from kudzu root, possesses the characteristic of neuroprotection, antioxidation and anticancer. In the present study, we observed that LPS induced over-production of nitric oxide (NO) and increased the level of intracellular ROS in N9 microglial cells, but it was inhibited by puerarin. Furthermore, treatment with puerarin on N9 cells suppressed the over-expression of inducible nitric oxide synthase (iNOS) induced by LPS which is implicated in intracellular O-linked $\beta$-N-acetylglucosamine (O-GlcNAc) level, phosphorylation of mitogen-activated protein kinase (MAPK) and nuclear factor $\kappa \mathrm{B}(\mathrm{NF}-\kappa \mathrm{B})$ signaling pathway. We also observed that the enhanced phosphorylation of p38, JNK and ERK1/2 in N9 cells induced by LPS were inhibited by puerarin, otherwise the down-regulation of O-GlcNAcylation level of protein in N9 cell induced by LPS was up-regulated by pretreatment with puerarin. These results indicate that puerarin effectively
\end{abstract}

Correspondence to: Dr Zhu Yang, Department of Obstetrics and Gynecology, the Second Affiliated Hospital of Chongqing Medical University, 76 Lin Jiang Road, Chongqing 400016, P.R. China

E-mail: paperyu@126.com

*Contributed equally

Abbreviations: NO, nitric oxide; iNOS, inducible nitric oxide synthase; ROS, reactive oxygen species; O-GlcNAc, O-linked $\mathrm{N}$-acetylglucosamine; MAPK, mitogen-activated protein kinase; $N F-\kappa B$, nuclear factor $\kappa B$

Key words: puerarin, inducible nitric oxide synthase, reactive oxygen species, microglial cell, mitogen-activated protein kinase, O-GlcNAcylation inhibits microglia activation induced by LPS through inhibiting expression of iNOS, production of NO and ROS which was mediated via regulating O-GlcNAcylation, phosphorylation of MAPK and NF- $\mathrm{KB}$ translocation.

\section{Introduction}

Microglial cells, macrophage-like cells in the central nervous system, participate in CNS immune response through releasing a variety of factors, including trophic factors and chemokines, to promote neuroprotection (1). Whereas under chronic inflammatory environment, excessive activation of microglia cells secrete cytotoxic substances and neurotoxic cytokines, such as reactive oxygen species (ROS) and nitric oxide (NO) which contribute to the pathogenesis of neurodegenerative diseases (2). Lipopolysaccharide (LPS), which consist in outer membrane of gram-negative bacteria as potent pro-inflammatory agents, induce microglial cell inflammatory injury through releasing proinflammatory mediators such as NO generated by inducible nitric oxide synthase (iNOS) $(3,4)$. $\mathrm{NO}$ is considered as a main risk factor of progressive damage in neurodegenerative diseases $(5,6)$. Microglia cell inflammatory response to LPS is involved in speedy regulation of many signal transduction molecules. The regulation of a signal pathway, such as mitogen-activated protein kinase (MAPK) and nuclear factor $\kappa \mathrm{B}(\mathrm{NF}-\mathrm{\kappa B})$, is implicated in major molecular mechanisms of LPS-induced NO and ROS production in microglial cells $(4,6-8)$.

O-Linked O-GlcNAcylation of protein, a single monosaccharide $\mathrm{N}$-acetylglucosamine (GlcNAc) attached to the hydroxyl groups of specific serine or threonine residues, is an important post-translational modification on nuclear and cytoplasmic proteins $(9,10)$. More than 500 proteins have been identified to be O-GlcNAcylated, and these proteins are involved in regulation of cell processes including cell cycle, transcription, trafficking and signaling (11). O-GlcNAc added to proteins is only catalyzed by O-GlcNAc transferase (OGT), and removal of O-GlcNAc from proteins by O-GlcNAc hexosaminidase (O-GlcNAcase). OGT is high expressed in macrophages, pancreas, and the nervous system (12). Some 
residues of proteins modified by O-GlcNAcylation are also phosphorylation sites, demonstrating a complex relationship between O-GlcNAcylation and phosphorylation which is the so-called 'yin-yang' hypothesis $(11,13)$. The interplay between O-GlcNAcylation and phosphorylation on functional proteins is pivotal to the aetiology of neurodegenerative disease (14). A major function of O-GlcNAc may be to antagonize phosphorylation, through competing with phosphorylation for a single site or proximal sites on proteins $(9,10,12)$. Evidence indicated that O-linked GlcNAc modification could modulate many transcription factors, including $\mathrm{NF}-\kappa \mathrm{B}$ involved in response to stress $(9,12,15)$.

Puerarin, an isoflavone is an active ingredient extracted from kudzu root, has been shown to possess neuroprotection, antioxidation and anticancer characteristics (16). It has been reported that puerarin regulated the inflammatory response induced by LPS via NF- $\kappa$ B pathway in THP-1 monocytederived macrophages (16). Recent studies show that NOS-induced production of NO was an important mediator in ischemic brain injury. However, the neuroprotective effect of puerarin is related to suppression of iNOS expression (17). Therefore, the present study was undertaken to investigate the molecular mechanisms of neuroprotection of puerarin, particularly anti-inflammatory effect of puerarin on microglial cell injuried by LPS through suppressing over-production of NO and expression of iNOS. The results demonstrated that puerarin effectively inhibit microglia activation by LPS via a series of signaling cascades mediated by phosphorylation or O-GlcNAcylation of functional proteins including MAPK.

\section{Materials and methods}

Chemicals and reagents. 3-(4,5-dimethylthiazol-2-yl)-2,5-diphenyltetrazolium bromide (MTT), LPS from Escherichia coli, dimethylsulfoxide (DMSO) and 2',7'-dichlorofluorescein diacetate (DCFH-DA) were purchased from Sigma (St. Louis, MO, USA). Puerarin was purchased from China institute for the control of drugs and biological products. 3-Amino,4aminomethyl-2',7'-difluorescein, diacetate (DAF-FM DA) were purchased from Beyotime Institute of Biotechnology (Jiangsu, China). Polyclonal rabbit or mouse antibodies against p38 MAPK, phospho-p38 (p-p38) MAPK, phospho-ERK1/2 (p-ERK1/2), JNK, phospho-JNK (p-JNK), O-ClcNAc, O-GlcNAc transferase (OGT), iNOS, Lamin B, horseradish peroxidase (HRP)-conjugated goat anti-rabbit $\operatorname{IgM}$ and horseradish peroxidase (HRP)-conjugated goat anti-rabbit or mouse IgG were purchased from Santa Cruz Biotechnology (Santa Cruz, CA, USA). ERK1/2 were purchased from Millipore. $\beta$-actin were purchased from Cell Signaling Technology. Polyclonal rabbit antibody against NF- $\kappa$ B was obtained from Beyotime Institute of Biotechnology (Jiangsu, China). Iscove's modified Dulbecco's medium (IMDM) and fetal bovine serum (FBS) were purchased from Gibco (Grand Island, NY, USA).

Cell culture and drug treatment. The murine microglial N9 cell line was a kind gift of Professor Yun Bai (the Third Military Medical University, Chongqing, China). The cell line was cultured in IMDM, supplemented with 10\% FBS,
$0.5 \% \beta$-mercaptoethanol, $1 \%$ glutamine, $100 \mathrm{U} / \mathrm{ml}$ penicillin, $100 \mathrm{U} / \mathrm{ml}$ streptomycin and $5 \mathrm{U} / \mathrm{ml}$ heparin. Cells were maintained in $95 \%$ air, $5 \% \mathrm{CO}_{2}$-humidified atmosphere at $37^{\circ} \mathrm{C}$. The cells were pre-treated with various concentrations of puerain for $24 \mathrm{~h}$ before stimulation. Puerarin was dissolved in dimethyl formamide (DMSO <0.1\%), which showed no deleterious effect on the viability of N9 cells in previous studies.

Cell viability assay with MTT. N9 cells were seeded in 96-well plates with $1.5 \times 10^{4}$ cells/well, treated with puerarin $(25,50,100$, $200 \mu \mathrm{M})$ or LPS $(1 \mu \mathrm{g} / \mathrm{ml})$. After being cultured for $24 \mathrm{~h}$, the medium was removed, cells were washed with PBS $(\mathrm{pH} 7.4)$ and incubated with MTT $(5 \mathrm{mg} / \mathrm{ml})$ in culture medium for $3 \mathrm{~h}$ at $37^{\circ} \mathrm{C}$. Then, culture medium was removed and the formazan blue was dissolved in $100 \mu \mathrm{l}$ of DMSO. The optical density was measured at $490 \mathrm{~nm}$ using a Sunrise Remote Microplate Reader (Grodlg, Austria).

NO measurement. Nitrite $\left(\mathrm{NO}^{2-}\right)$, converted from $\mathrm{NO}$ and molecular oxygen, reflects extracellular NO release from activated N9 cells. N9 cells were plated in 24-well plates and pretreated with puerarin for $24 \mathrm{~h}$, then treated with LPS for $20 \mathrm{~h}$. The culture medium was recovered for Griess assay. The required volume of culture medium $(50 \mu \mathrm{l})$ was mixed with an equal (1:1) volume of Griess reagent (1\% sulfanilamide and $0.1 \% \mathrm{~N}$-naphthylethyl-ethylenediamine dihydrochloride in $5 \%$ phosphoric acid) for $10 \mathrm{~min}$ at $37^{\circ} \mathrm{C}$ in the dark. The absorbance was assessed at $540 \mathrm{~nm}$ using a Sunrise Remote Microplate Reader. A standard curve was generated using $\mathrm{NaNO}_{2}$ in each experiment for quantification.

Intracellular generation of $\mathrm{NO}$ was measured by flow cytometry. Cells were seeded into 6-well plates and pretreated with puerarin for $24 \mathrm{~h}$. Cells were washed and pretreated with puerarin for $24 \mathrm{~h}$, then treated with LPS for $20 \mathrm{~h}$. The cells were washed twice with PBS and loaded with DAF-FM DA (dilution 1:1000) for $30 \mathrm{~min}$ at $37^{\circ} \mathrm{C}$ in the dark. The cells were washed twice, resuspended in PBS and measured with flow cytometry.

Intracellular ROS measurement with confocal immunofluorescence microscopy. Cells were seeded into 6-well plates and pretreated with puerarin and/or LPS for $24 \mathrm{~h}$. The cells were washed with PBS and loaded with DCFH-DA $(20 \mathrm{mM})$ for $1 \mathrm{~h}$ at $37^{\circ} \mathrm{C}$ in the dark. Cells were washed twice with PBS and analysed using Leica TCS-SP2 microscope (Wetzlar, Germany).

Western blot analysis. Total cell proteins were extracted as follows: cells were washed twice and resuspended in ice-cold PBS, then centrifuged at $600 \mathrm{x} \mathrm{rpm}$ for $10 \mathrm{~min}$ at $4^{\circ} \mathrm{C}$. Cells were lysed with $200 \mu 1$ RIPA lysis buffer (50 mM Tris with $\mathrm{pH} 7.4,150 \mathrm{mM} \mathrm{NaCl}, 1 \%$ TritonX-100, $1 \%$ sodium deoxycholate, $0.1 \%$ SDS and $0.05 \mathrm{mM}$ EDTA), were vortexed every $5 \mathrm{~min}$ at $4^{\circ} \mathrm{C}$ for $30 \mathrm{~min}$. The cell suspension was centrifuged at $12,000 \mathrm{x} \mathrm{g}$ for $15 \mathrm{~min}$ at $4^{\circ} \mathrm{C}$ and the supernatant was collected as total cell extracts. Cell cytoplasm and nuclei proteins were separated with a nuclear and cytoplasmic protein extraction kit (Beyotime, Jiangsu, China), according 
to manufacturer's instructions. Protein concentration was quantified by the bicinchoninic acid protein assay kit (Biomed Biotech Co., Ltd., Beijing, China). All samples were stored at $-80^{\circ} \mathrm{C}$ until further analysis.

Before Western blot analysis, protein was boiled in $5 \mathrm{X}$ loading buffer (125 mM Tris- $\mathrm{HCl}, \mathrm{pH} 6.8,10 \%$ SDS, $8 \%$ dithiothreitol, $50 \%$ glycerol and $0.5 \%$ bromochlorophenol blue) for $10 \mathrm{~min}$. Aliquots of $30 \mu \mathrm{g}$ of protein was separated by 6\%-12\% SDS-PAGE and transferred on to a PVDF membrane. The membrane was blocked by $5 \%$ non-fat dry milk in PBS with $0.1 \%$ Tween 20 (PBST) for $1 \mathrm{~h}$, or $1.5 \%$ BSA in TBS with $0.1 \%$ Tween 20 (TBST) for at least $2 \mathrm{~h}$, then incubated with the primary antibodies overnight at $4^{\circ} \mathrm{C}$. The immunoblots were washed five times $(3 \times 10 \mathrm{~min})$ in PBST or TBST, then incubated with a corresponding secondary horseradish peroxidase-conjugated IgG (for iNOS, NF- $\kappa$ B, p-p38, p38, p-ERK1/2, ERK1/2, p-JNK, OGT, lambin B and $\beta$-actin blots) and $\mathrm{IgM}$ (for O-GlcNAc blot) for $1 \mathrm{~h}$. After washing with PBST or TBST, the blots were reacted with enhanced chemiluminescence reagents (ECL). Densitometric analysis was performed with the use of 720 BK/01837 System (Bio-Rad, Hercules, CA, USA).

RNA isolation and quantitative real-time PCR analysis. Total RNA was isolated from treatment N9 cells using cold Trizol reagent (Takara, Dalian, China) and RNA (500 ng) was reverse transcribed using RT reagent kit (Takara) according to the manufacturer's protocol. The primer pair sequences for amplifying iNOS and GAPDH were: iNOS, sense, 5'-TGG AGC GAG TTG TGG ATT GTC-3', antisense, 5'- CCC TTT GTG CTG GGA GTA GT-3'; GAPDH, sense, 5'-CAA GGT CAT CCA TGA CAA CTT TG-3', antisense, 5'-GGC CAT CCA CAG TCT TCT GG-3'. The reaction system contains $5 \mu 1$ SYBR green mix, sense and antisense primers each $30 \mu \mathrm{M}$, cDNA $1 \mu \mathrm{l}$, and RNA-free $\mathrm{H}_{2} \mathrm{O}$ to $10 \mu \mathrm{l}$. Real-time PCR was performed with the follwing reaction parameters: $3 \mathrm{~min}$ at $95^{\circ} \mathrm{C}, 30$ cycles followed for $10 \mathrm{sec}$ at $95^{\circ} \mathrm{C}, 30 \mathrm{sec}$ at $60^{\circ} \mathrm{C}$, then $65^{\circ} \mathrm{C}-95^{\circ} \mathrm{C}$ to dissolve. The amount of target normalized to $\beta$-actin as an endogenous reference and relative to the control was by $2^{-\Delta \Delta C t}$.

Transient transfection and $N F-\kappa B$ reporter assays. N9 cells were seeded in 24 -well plates with $7.5 \times 10^{4}$ cells/well, and co-transfected with $0.4 \mu \mathrm{g} \mathrm{NF- \kappa B}$ plasmid (with firefly luciferase) and $0.4 \mu \mathrm{g}$ pRL-TK plasmid (with Renilla luciferase used as internal control for transfection efficiency) using Lipofectamine reagent (Invitrogen) for $6 \mathrm{~h}$. Twenty-four hours after transfection, cells were washed and treated with purearin $(25,100 \mu \mathrm{M})$ for $24 \mathrm{~h}$. Luciferase activity was determined with the Dual-Luciferase reporter assay system (Promega). Firefly/Renilla represented relative luciferase activity.

Statistics. Statistical evaluation were performed using SPSS 10.0 package (SPSS Inc., Chicago, IL, USA). Data are reported as mean \pm SD of 3-5 independent experiments. One-way ANOVA was used to analyze statistical differences between multiple groups, two groups were compared using the Student's t-test followed by a Bonferroni correction. Values of $\mathrm{P}<0.05$ were considered to be statistically significant.

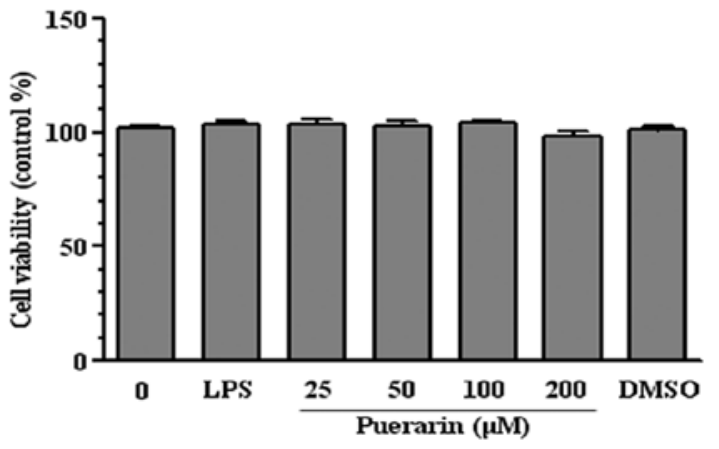

Figure 1. Effect of puerarin or LPS on N9 cell viability. Cells were treated with puerarin $(25,50,100$ and $200 \mu \mathrm{M})$, LPS $(1 \mu \mathrm{g} / \mathrm{ml})$ or DMSO for $24 \mathrm{~h}$ separately. Then, cell viability was measured by MTT assay. Data are expressed as means \pm SD of three independent experiments.

\section{Results}

The viability loss in N9 cells is impacted by both puerarin and $L P S$. We first carried out the studies of viability loss in N9 cells induced by LPS, used as proinflammatory factor in injury cell model, and treated with puerarin which was used as protective agent in the present model. After treatment with LPS for $20 \mathrm{~h}$ or various concentrations of puerarin for $24 \mathrm{~h}$ separately, cell viability was examined by MTT method. As shown in Fig. 1, discrepant loss of cell viability was not observed with used concentrations of LPS or puerarin versus the control groups. Results show that treatment with concentration of puerarin (25-100 $\mu \mathrm{M})$ for $24 \mathrm{~h}$ or LPS $(1 \mu \mathrm{g} / \mathrm{ml})$ for $24 \mathrm{~h}$ had no significant cytotoxic effect on N9 cell viability. So, concentrations of LPS and puerarin or vehicle were used in the extensive study.

Puerarin inhibits production of NO and ROS in N9 cells induced by LPS. Microglial N9 cells by LPS-stimulation activate release of $\mathrm{NO}$, which mediates inflammatory processes in CNS (18). To investigate potential neuroprotection of puerarin on microglia activation, we examined the effect of puerarin on the production of extracellular NO by Griess assay and intracellular NO by flow cytometry. As shown in Fig. 2A, N9 cells stimulated with LPS for $20 \mathrm{~h}$ increased extracellular NO production significantly. When N9 cells were co-treated with puerarin $(25,50,100 \mu \mathrm{M})$ and LPS, extracellular NO levels were significantly decreased. Therefore, we chose puerarin $(25,100 \mu \mathrm{M})$ in the subsequent experiments. Similar to the change with extracellular NO, LPS increased intracellular NO producetion. High concentration of puerarin $(100 \mu \mathrm{M})$ repressesed the production of intracellular NO induced by LPS (Fig. 2B). Results suggested that puerarin had a potent inhibitory activity on NO production in N9 cells induced by LPS.

Activated N9 cells participate in the local cell-mediated immunity, produce neurotoxicants such as NO inducing ROS generation to mediate oxidative injury $(19,20)$. It has been reported that free oxygen radicals released by microglia have a neurotoxic effect in co-cultures of neurones and microglia $(19,20)$. To determinate neuroprotection of puerarin, the effect of puerarin on intracellular ROS production was evaluated by confocal immunofluorescence microscopy. As shown in Fig. 3, treatment with LPS increased fluorescence intensity 

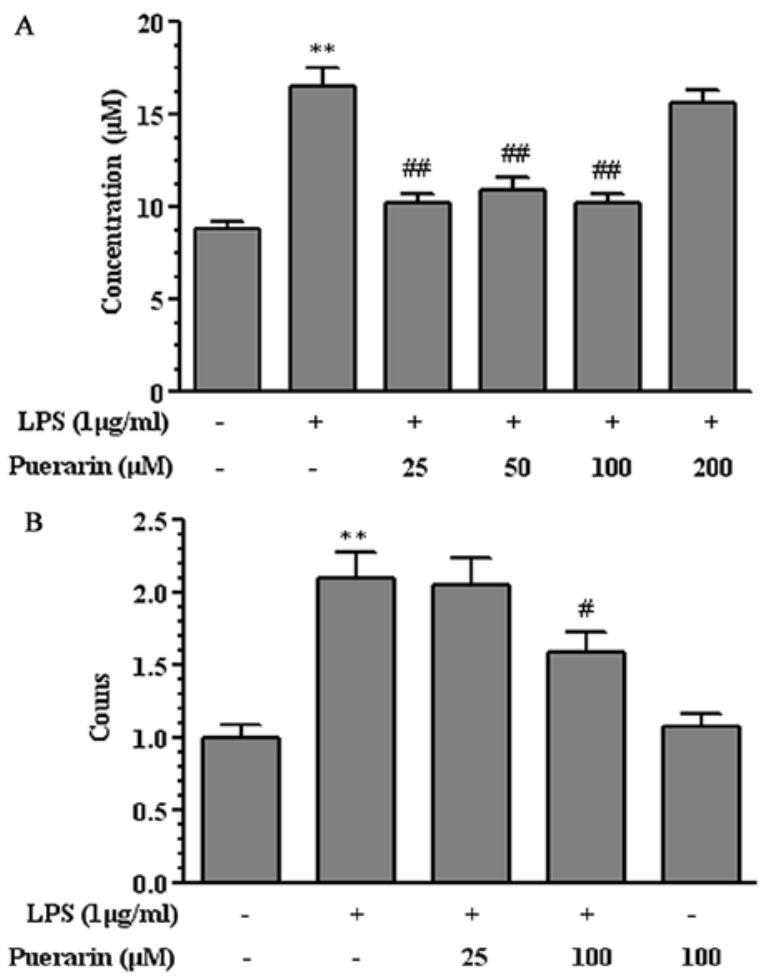

Figure 2. Effect of puerarin on NO production in N9 cells induced by LPS. (A) Microglia were pretreated with puerarin $(25,50,100$ and $200 \mu \mathrm{M})$ for $24 \mathrm{~h}$, then treated with LPS $(1 \mu \mathrm{g} / \mathrm{ml})$ for $20 \mathrm{~h}$. The culture medium was collected for the Griess assay. (B) Microglia were pretreated with puerarin (25 and $100 \mu \mathrm{M})$ for $24 \mathrm{~h}$, then treated with LPS $(1 \mu \mathrm{g} / \mathrm{ml})$ for $20 \mathrm{~h}$. Then, intracellular NO was tested by loading with DAF-FM DA for $30 \mathrm{~min}$ and cells were washed and resuspended with PBS for flow cytometry. ${ }^{* *} \mathrm{P}<0.01$ compared to the vehicle-treated group. ${ }^{\#} \mathrm{P}<0.05,{ }^{\# \#} \mathrm{P}<0.01$ compared to the LPS-treated group. in N9 cell (Fig. 3B). The increase could be reduced by pretreatment with puerarin (Fig. 3C and D). This suggested that puerarin had an inhibitory effect on LPS-induced ROS generation.

Puerarin inhibits expression of iNOS at transcription and translation levels in N9 cells induced by LPS. NOS include three member enzymes, specifically inducible NO synthase (iNOS) which is produced mainly by activated glial cells, participate in NO production from L-arginine (21). The synthesis of iNOS along with the release of NO by microglia is associated with the progression of neurodegeneration $(4,22)$. In this study, we demonstrated that puerarin was able to inhibit LPS-induced NO production. Next, we examined whether the suppression of NO production by puerarin was due to downregulation of iNOS.

N9 cells were pre-treated with puerarin $(25$ and $100 \mu \mathrm{M})$ for $24 \mathrm{~h}$, then stimulated with LPS $(1 \mu \mathrm{g} / \mathrm{ml})$ for $20 \mathrm{~h}$. Extracted protein was measured by Western blot analysis. As shown in Fig. 4A, LPS-stimulated N9 cells increased the protein level of iNOS. Pre-treatment with puerarin significantly suppressed iNOS protein production in a dosedependent manner.

The effects of puerarin on iNOS mRNA expression was analysed by quantitative real-time PCR. Similar results to the protein level is shown in Fig. 4B, compared with the LPS-treated group, puerarin pre-treatment significantly attenuated iNOS mRNA expression. These results indicated that puerarin inhibited NO production via suppressing iNOS at transcription and translation levels.
A

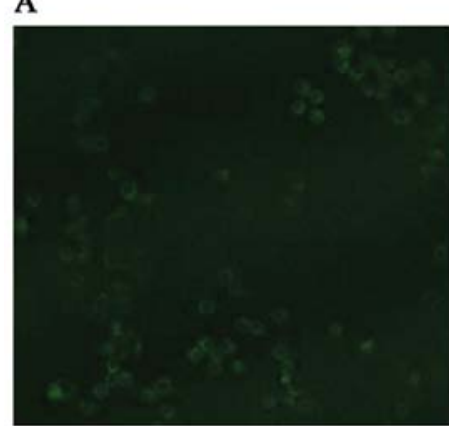

D

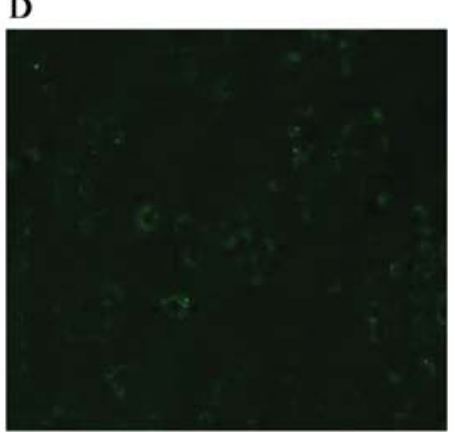

B

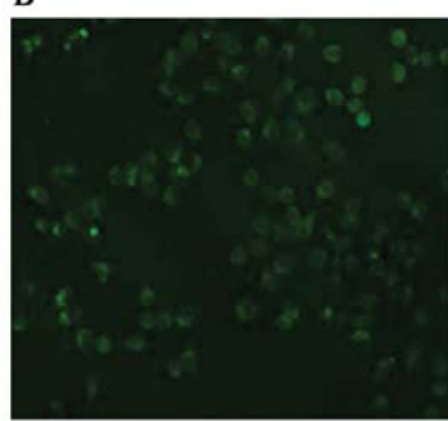

$\mathrm{E}$

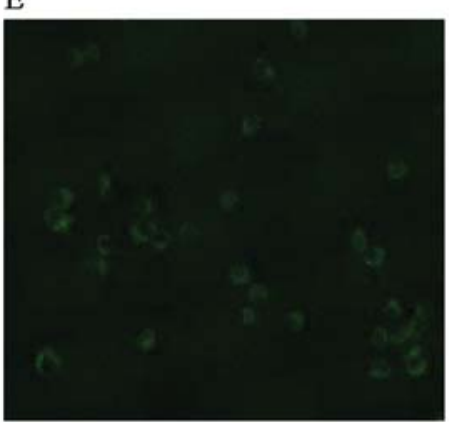

Figure 3. Effect of puerarin on ROS induced by LPS in N9 cells. Cells were pre-incubated with puerarin ( 25 and $100 \mu \mathrm{M})$ for 24 h, then exposed to LPS $(1 \mu \mathrm{g} / \mathrm{ml})$ for $20 \mathrm{~h}$. The cells were washed with PBS and loaded with DCFH-DA for $1 \mathrm{~h}$. Then, measured by Confocal immunofluorescence microscopy. (A) the vehicle-treated group; (B) the LPS group; (C) the LPS plus puerarin (25 $\mu \mathrm{M})$ group; (D) the LPS plus puerarin (100 $\mu \mathrm{M})$ group; (E) the puerarin (100 $\mu \mathrm{M})$ group. Scale bar, $75 \mu \mathrm{m}$. 
A
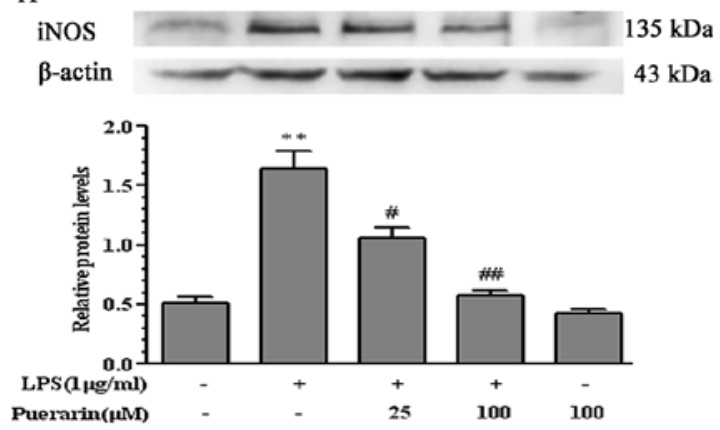

B

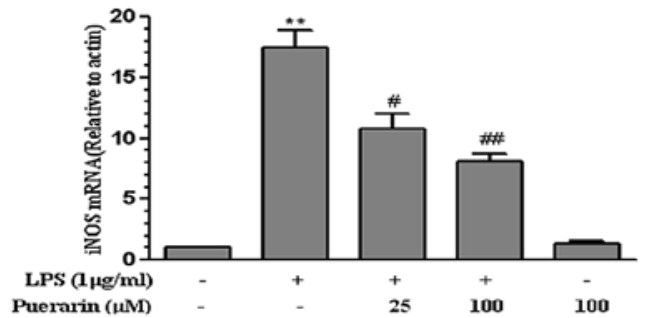

Figure 4. The effect of puerarin on expression of iNOS protein (A) and mRNA (B) in N9 cells. Cells were pretreated with puerarin $(25$ and $100 \mu \mathrm{M})$ for $24 \mathrm{~h}$ before stimulation with LPS $(1 \mu \mathrm{g} / \mathrm{ml})$ for another $20 \mathrm{~h}$ to test iNOS protein with Western blot analysis, or $6 \mathrm{~h}$ to measure mRNA with real-time PCR. ${ }^{* *} \mathrm{P}<0.01$ compared to the vehicle-treated group. ${ }^{\#} \mathrm{P}<0.05,{ }^{\# \#} \mathrm{P}<0.01$ compared to the LPS-treated group.

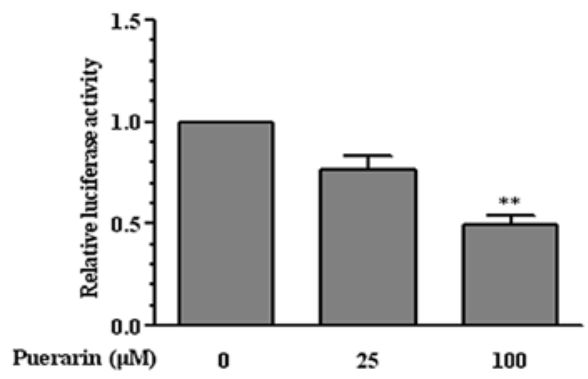

Figure 5. Effect of puerarin on NF- $\kappa \mathrm{B}$ activation in N9 cells. Cells were cotransfected with the NF- $\kappa \mathrm{B}$ and pLR-TK plasmid using Lipofectamine 2000 and incubated with puerarin $(25$ and $100 \mu \mathrm{M})$ for $24 \mathrm{~h}$. the luciferase reporter construct possessed consensus NF- $x \mathrm{~B}$ and pLR-TK with puerarin $(0 \mu \mathrm{M})$ binding sites. ${ }^{* *} \mathrm{P}<0.01$ compared to the control.
Puerarin exhibited potent inhibition on activation and translocation of $N F-\kappa B$ in N9 cells induced by LPS. NF- $\mathrm{B}$ is an important transcription factor that regulates the transcription of many genes associated with inflammation, including the iNOS and ROS relative gene $(4,18,21,23)$. Resting NF- $\kappa$ B is located in the cytoplasm, $N F-\kappa B$ rapidly transfers to the nucleus in response to stimuli and activates the transcription of proinflammation (23). To determine whether activation of $\mathrm{NF}-\kappa \mathrm{B}$ was suppressed by puerarin, we first analyzed luciferase reporter constructs containing the $\mathrm{NF}-\kappa \mathrm{B}$ promoter, which was markedly inhibited in N9 cells after puerarin (25 and $100 \mu \mathrm{M}$ ) treatment for $24 \mathrm{~h}$ (Fig. 5). In addition, we performed the NF- $\kappa \mathrm{B}$ translocation experiment by Western blot analysis. N9 cells were pretreated with puerarin (25 and $100 \mu \mathrm{M}) 24 \mathrm{~h}$, followed by LPS $(1 \mu \mathrm{g} / \mathrm{ml})$ incubation for $6 \mathrm{~h}$. As shown in Fig. 6, LPS induced NF- $\kappa$ B to translocate from cytoplasm into nucleus. In contrast, treatment with puerarin significantly inhibited the translocation of $\mathrm{NF}-\kappa \mathrm{B}$ in $\mathrm{N} 9$ cells. These data indicated that puerarin $(100 \mu \mathrm{M})$ effectively attenuated the $\mathrm{NF}-\kappa \mathrm{B}$ activation and translocation in N9 cells.

Puerarin represses phosphorylation of some of MAPKs in N9 cells induced by LPS. Activated NF- $\mathrm{BB}$ and MAPKs participate in production of NO and iNOS induced by LPS $(4,7,24)$. MAPK regulated NO and iNOS production has been proved to require $\mathrm{NF}-\kappa \mathrm{B}$ activation in glial cells $(24,25)$. The subsequent experiments were designed to examine the effect of puerarin on phosphorylation of MAPK. N9 cell was pretreated with puerarin ( 25 and $100 \mu \mathrm{M}$ ) for $24 \mathrm{~h}$, then exposed to LPS $(1 \mu \mathrm{g} / \mathrm{ml})$ for $30 \mathrm{~min}$. As shown in Fig. 7, treatment with LPS induced high phosphorylation of certain kinases of MAPK, including p38, ERK and JNK. The enhanced phosphorylation of p38 and JNK in N9 cell induced by LPS were inhibited by puerarin at higher concentration $(100 \mu \mathrm{M})$. Both concentration of puerarin at $25 \mu \mathrm{M}$ and $100 \mu \mathrm{M}$ inhibited phosphorylation of ERK1/2 in N9 cells induced by LPS. In our previous study, the increase of MAPKs was detected after 15 min of LPS stimulation, reached a maximum after $30 \mathrm{~min}$, and then decreased. It was shown that the activation of MAPKs is before the activation of $\mathrm{NF}-\kappa \mathrm{B}(26)$. These data demonstrated
A

$\mathrm{NF}-\kappa \mathrm{B}(\mathrm{CE})$

$\beta$-actin

$-$

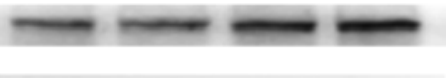

$65 \mathrm{kDa} \quad \mathrm{NF}-\mathrm{kB}(\mathrm{HE})$
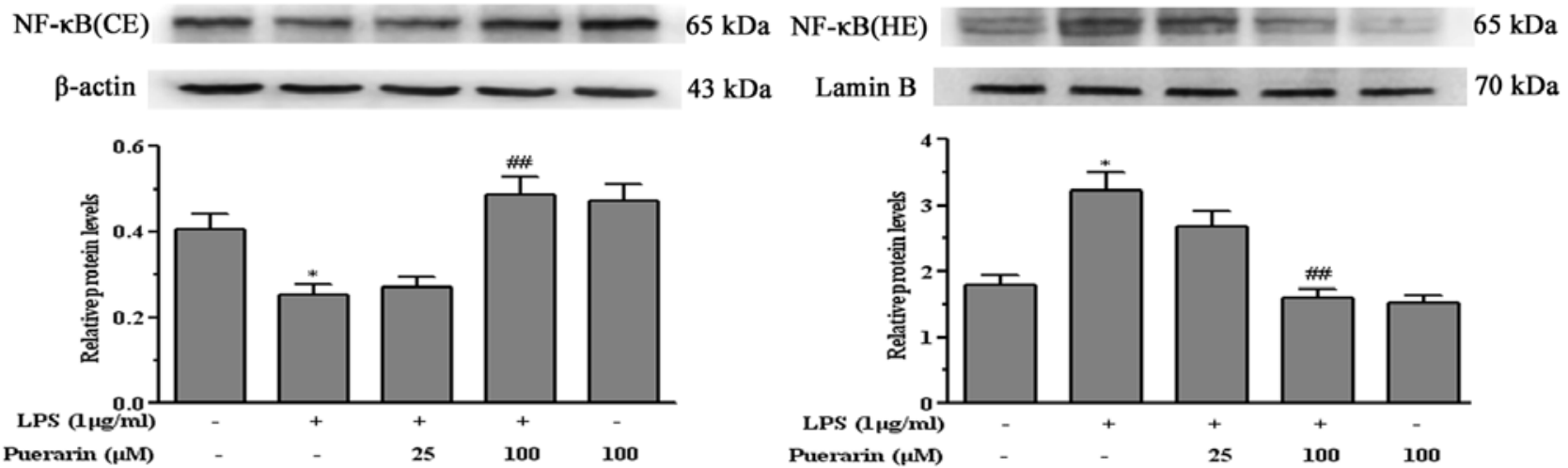

B $43 \mathrm{kDa}$

\section{$70 \mathrm{kDa}$}

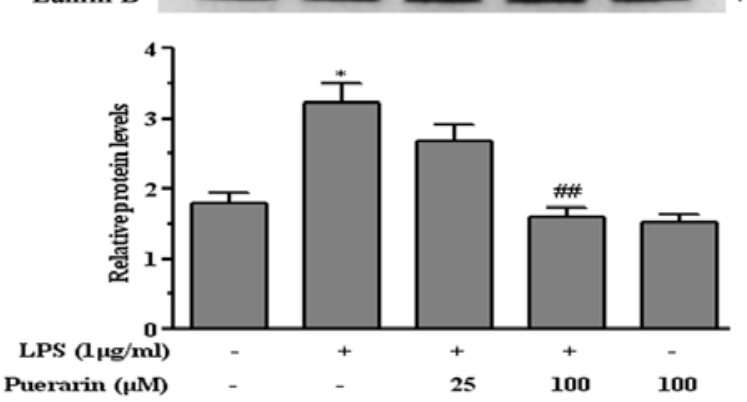

Figure 6. The effects of puerarin on the translocation of NF- $\kappa \mathrm{B}$ in N9 cells. Cells were pretreated with puerarin (25 and $100 \mu \mathrm{M})$ for $24 \mathrm{~h}$, then stimulated with LPS $(1 \mu \mathrm{g} / \mathrm{ml})$ for $6 \mathrm{~h}$. Cell cytoplasm and nuclei proteins were extracted with a nuclear and cytoplasmic protein extraction kit. CE, cytoplasmic extracts; NE, nuclear extracts. ${ }^{*} \mathrm{P}<0.05$ compared to the vehicle-treated group. ${ }^{\# \prime} \mathrm{P}<0.01$ compared to the LPS-treated group. 
A
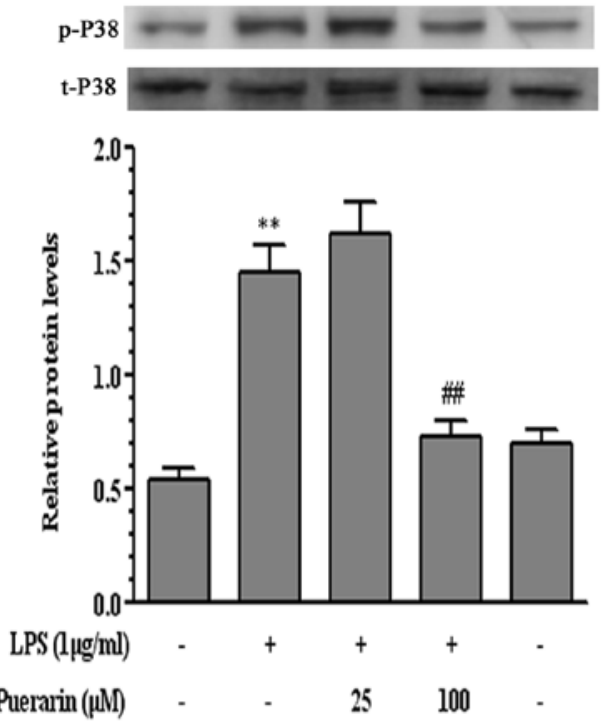

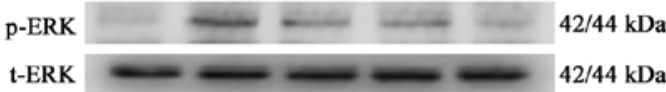

$38 \mathrm{kDa}$

$38 \mathrm{kDa}$
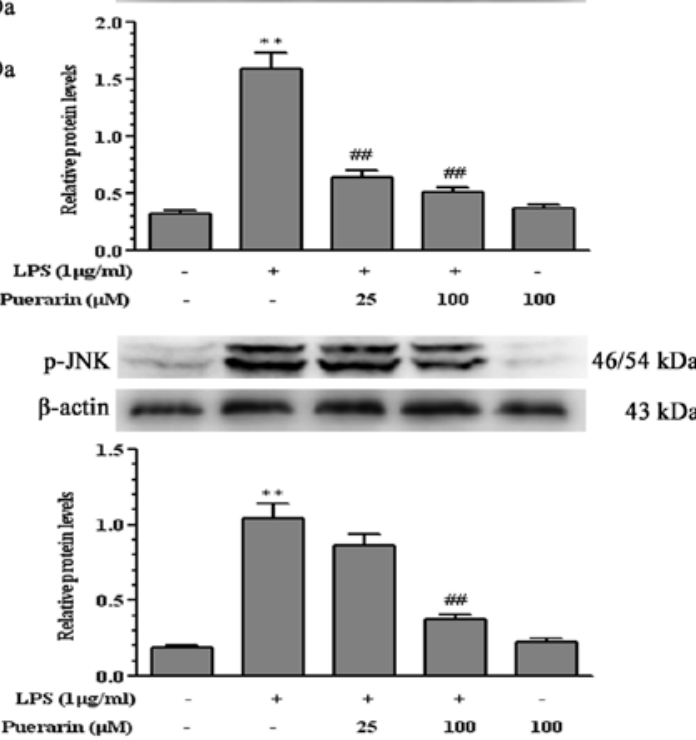

Figure 7. The effects of puerarin on the activation of MAPKs, including (A) p38 MAPK (B) ERK1/2 and (C) JNK. Cells were pretreated with puerarin (25 and $100 \mu \mathrm{M})$ for $24 \mathrm{~h}$ and then stimulated with LPS $(1 \mu \mathrm{g} / \mathrm{ml})$ for $30 \mathrm{~min}$. After treatment, cell lysates were prepared to evaluate the protein levels of phosphorylated p38 MAPK (p-p38), total p38 MAPK (t-p38), phosphorylated ERK1/2 (p-ERK1/2), total ERK1/2 (t-ERK1/2), phosphorylated JNK (p-JNK) and $\beta$-actin were determined by Western blot analysis. ${ }^{* *} \mathrm{P}<0.01$ compared to the vehicle-treated group. ${ }^{\# \#} \mathrm{P}<0.01$ compared to the LPS-treated group.

A

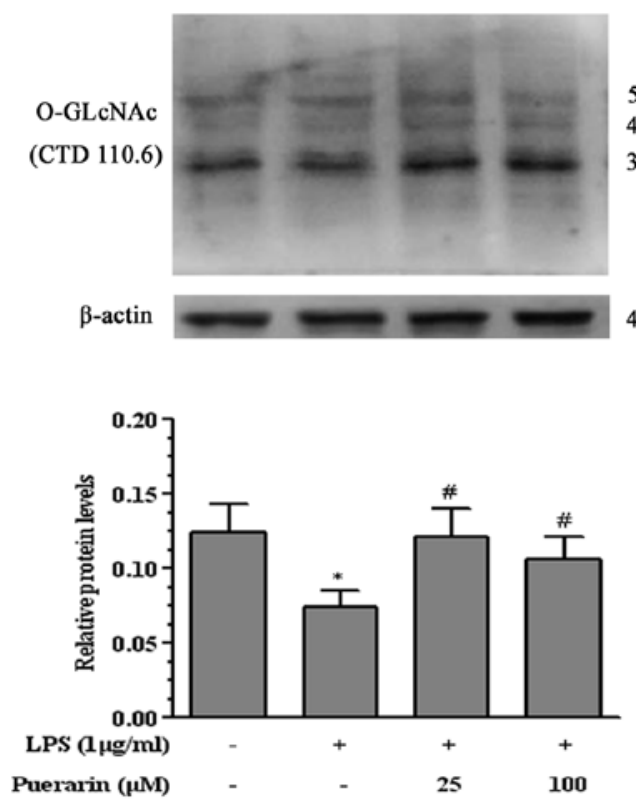

B

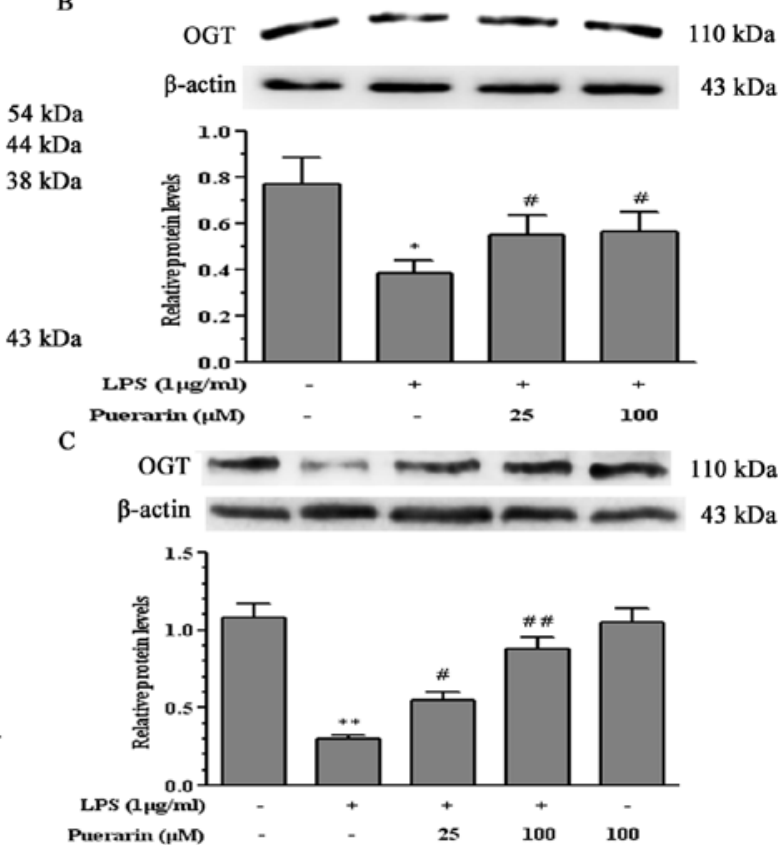

Figure 8. The effect of Puerarin on O-GlcNAc in N9 cells. (A and B) Cells were pretreated with puerarin (25 and $100 \mu \mathrm{M})$ for $24 \mathrm{~h}$, then stimulated with LPS $(1 \mu \mathrm{g} / \mathrm{ml})$ for $30 \mathrm{~min}$. Extracted protein to measure O-GlcNAc and OGT by Western blot analysis. (C) Cells were pretreated with puerarin (25 and $100 \mu \mathrm{M})$ for $24 \mathrm{~h}$ and then treated with LPS $(1 \mu \mathrm{g} / \mathrm{ml})$ for $6 \mathrm{~h} .{ }^{*} \mathrm{P}<0.05,{ }^{* *} \mathrm{P}<0.01$ compared to the vehicle-treated group. ${ }^{*} \mathrm{P}<0.05,{ }^{\# \#} \mathrm{P}<0.01$ compared to the LPS-treated group.

that puerarin down-regulates NF- $\mathrm{B}$ through suppression of MAPKs phosphorylation.

Puerarin up-regulates OGT expression and reverses the decrease of $O$-GlcNAcylation on protein in LPS-induced microglia N9 cells. O-GlcNAc, a post-translational modifica- tion of nuclear and cytoplasmic proteins resembling protein phosphorylation (27) plays an active role in neurodegenerative diseases $(12,14,28)$ which is involved in modulating many transcription factors (29) and signal transduction pathways (30). The relationship between O-GlcNAc and phosphorylation which has been referred to as the 'yin-yang' 
hypothesis, is a complex interplay including competition to occupy a single site or proximal sites on proteins $(11,27)$. The attachment of O-GlcNAc to proteins is catalyzed by O-linked N-acetylglucosamine (GlcNAc) transferase (OGT), which is widely expressed in macrophages, pancreas, and the nervous system (12). Thus, we examined the effect of puerarin on O-GlcNAc level and evaluated whether the change related to MAPKs and NF- $\kappa \mathrm{B}$ pathway. As shown in Fig. 8, LPS-treatment for $30 \mathrm{~min}$ down-regulated the O-GlcNAc level (Fig. 8A), which is potentially relative in up-regulated phosphorylation of p38, ERK and JNK in N9 cells (Fig. 6). Pretreatment with puerarin $(25$ and $100 \mu \mathrm{M})$ for $24 \mathrm{~h}$ was able to reverse down-regulation O-GlcNAc level in N9 cells induced by LPS. The changes in OGT paralleled with O-GlcNAc level (Fig. 8B) in N9 cells after exposure to LPS for $30 \mathrm{~min}$. The effect of puerarin on O-GlcNAcylation in N9 cells was not dose-dependent after treating with LPS for 30 min. Furthermore, after being treated with LPS for $6 \mathrm{~h}$, expression of OGT in N9 cells was decreased significantly (Fig. 8C) and puerarin increased expression of OGT in a dosedependent manner. The increasing translocation of $\mathrm{NF}-\kappa \mathrm{B}$ was observed in a previous test (Fig. 5). These date suggested that puerarin exerted reversal of the decrease of O-GlcNAc level induced by LPS, associated with down-regulated phosphorylation of MAPKs and NF- $\kappa$ B signaling pathway. The relation between O-GlcNAcylation and phosphorylation of MAPKs impacted by puerarin in N9 cells induced by LPS requires further study.

\section{Discussion}

Microglia, macrophage-like cells, are ubiquitously distributed in the central nervous system (CNS). In general, microglial cells are quiescent (31) activated microglia in the vicinity of neuronal injury are involved in the removal of infectious agents and damaged cells from degenerating neurons (32) can contribute to tissue repair and neuroprotection (2). On the other hand, pathologic microglial excessive activation participate in the occurrence and development of neurological disorders (33). Chronic neuro-inflammation results primarily in the activation of microglia, excessive release of a number of potentially neurotoxic cytokines, leading to further activation of microglia, causing most neurodegenerative diseases, such as Alzheimer disease and Parkinson disease $(2,34,35)$. Microglial cells are activated in response to environmental stress and stimulation with LPS (21). Thus, control of the microglial over-reaction and the inflammatory response has attracted great attention. Puerarin, a major isoflavonoid extract from kudzu root, has potent neuroprotective effect against brain injury through the inhibition of inflammatory responses (36). Therefore, in the present study, we wanted to determine the mechanism of neuroprotective effect of puerarin on inflammatory response to LPS through protecting microglia from excessive activation.

NO, a free radical, acts as a neurotoxin or signaling molecule involved in the immune, cardiovascular and nervous systems. Over-production of NO is synthesized by inducible nitric oxide synthase (iNOS) (37) which has a neurotoxic effect (17). It has been discovered that LPS promote microglial activation, releasing NO or cytokines, which mediate inflammatory injury (38). In previous studies, puerarin decreased NO production (39) and inhibited the activity of iNOS (17). These findings are quite similar to ours. Our study shows that puerarin pretreatment inhibited NO production, and blocked iNOS protein level through the inhibition of iNOS transcription in LPS-induced N9 cells. Incubation of N9 cells with puerarin at various concentrations $(25,50,100,200 \mu \mathrm{M})$ or LPS $(1 \mu \mathrm{g} / \mathrm{ml})$ for $24 \mathrm{~h}$ showed no significant toxicity on cell viability, suggesting that the influence of puerarin or LPS on NO and iNOS levels was not due to altered cell numbers.

$\mathrm{NF}-\kappa \mathrm{B}$, a transcription factor, is believed to be a critical regulator in inflammatory and immune responses (40). NF- $\kappa \mathrm{B}$, a dimer component of p65 (RelA) and p50 subunits in the cytoplasm, binds the inhibitor I $\mathrm{B}$ (12). LPS induce I- $\kappa \mathrm{B}$ phosphorylating, subsequent ubiquitinating, degradating, then dissociating from the $\mathrm{NF}-\kappa \mathrm{B} / \mathrm{I} \kappa \mathrm{B}$ complex $(4,37)$. The liberated $N F-\kappa B$ then translocates from the cytoplasm to the nucleus and activates the transcription of proinflammatory factors, such as NO, iNOS $(4,40)$. It has been recognized that posttranslational modifications involve the activation of $\mathrm{NF}-\kappa \mathrm{B}$, including phosphorylation and acetylation $(9,12)$. $\mathrm{O}-\mathrm{GlcNAc}$ modifies NF- $\mathrm{B}$ via disrupting its interaction with $\mathrm{I} \kappa \mathrm{B}$, which results in translocation of $\mathrm{NF}-\kappa \mathrm{B}$ into the nuclear and stimulation of NF- $\kappa \mathrm{B}$-dependent gene expression $(40,41)$. Our study revealed that, LPS encouraged NF- $\kappa$ B translocation into the nucleus, and the enhancement was significantly inhibited by puerarin at higher concentrations. Regulation of NO include two critical signal transduction molecules, MAPK and NF- $x \mathrm{~B}(4,6,8)$. It has been documented that MAPKs, such as p38, JNK, and ERK, are major modulators for iNOS expression induced by LPS in microglial cells $(6,7)$. MAPKs modulate iNOS expression via up-regulating NF- $\kappa \mathrm{B}$ activity $(6,7)$. In our previous study, the increase of MAPKs was detected after 15 min of LPS stimulation, reached a maximum after $30 \mathrm{~min}$, and then decreased. It was shown that the activation of MAPKs is before the activation of $\mathrm{NF}-\kappa \mathrm{B}$. In this study we found that puerarin has suppressive effect on MAPK phosphorylation.

O-linked $\beta$-N-acetylglucosamine (O-GlcNAc), a highly dynamic post-translational modification, which modify nuclear and cytoplasmic proteins, plays a key role in signal transduction pathways (15). It has been suggested that O-linked GlcNAcylation and phosphorylation are critical posttranslational modifications $(9,12)$. Many O-GlcNAcylation modified sites are also phosphorylation sites, demonstrating a complex relationship between O-GlcNAcylation and phosphorylation, the so-called 'yin-yang' hypothesis $(11,13)$. O-GlcNAcylation has a major function of antagonizing phosphorylation, through competing with phosphorylation for sites on proteins $(9,10,12)$. Increasing O-GlcNAc levels inhibit the inflammatory response during resuscitation of trauma-hemorrhage models (15) and negatively correlate with the expression of phosphorylation of p38 MAPK and NF- $\mathrm{B}$ (42). In anti-inflammatory processes, inhibition of NF- $\kappa \mathrm{B}$ signaling may increase O-GlcNAc levels (15). Our studies show that puerarin exerted inhibition of the decrease of O-GlcNAc and OGT level induced by LPS, associated with down-regulated MAPKs and NF- $\mathrm{B}$ signaling pathway. 
In conclusion, our findings demonstrated that puerarin had an inhibitory effection on over-production of NO and iNOS in LPS-induced N9 cells. The molecular mechanism include increasing the O-GlcNAc level, attenuating the MAPK phosphorylation, inhibiting NF- $\mathrm{BB}$ activation, and suppressing intracellular ROS formation. Our results reveal that puerarin as a potential therapeutic agent for use in the treatment of neurodegenerative diseases.

\section{Acknowledgements}

We are grateful for financial support from National Nature Science Foundation of China (Project no. 81070222) and Nature Science Foundation of Chongqing (Project no. CSTC, 2009BA5083).

\section{References}

1. Zhao TZ, Xia YZ, Li L, et al: Bovine serum albumin promotes IL-1beta and TNF-alpha secretion by N9 microglial cells. Neurol Sci 30: 379-383, 2009.

2. Bodrato N, Franco L, Fresia C, et al: Abscisic acid activates the murine microglial cell line N9 through the second messenger cyclic ADP-ribose. J Biol Chem 284: 14777-14787, 2009.

3. Yuan D, Ma B, Yang JY, et al: Anti-inflammatory effects of rhynchophylline and isorhynchophylline in mouse N9 microglial cells and the molecular mechanism. Int Immunopharmacol 9 : 1549-1554, 2009.

4. Ha SK, Moon E and Kim SY: Chrysin suppresses LPS-stimulated proinflammatory responses by blocking NF-kappaB and JNK activations in microglia cells. Neurosci Lett 485: 143-147, 2010.

5. Chang LC, Tsao LT, Chang CS, et al: Inhibition of nitric oxide production by the carbazole compound LCY-2-CHO via blockade of activator protein-1 and CCAAT/enhancer-binding protein activation in microglia. Biochem Pharmacol 76: 507-519, 2008.

6. Platten M, Eitel K, Wischhusen J, Dichgans J and Weller M: Involvement of protein kinase Cdelta and extracellular signalregulated kinase- 2 in the suppression of microglial inducible nitric oxide synthase expression by $\mathrm{N}-[3,4-$ dimethoxycinnamoyl] anthranilic acid (tranilast). Biochem Pharmacol 66: 1263-1270, 2003.

7. Bi W, Zhu L, Wang C, Liang Y, Liu J, Shi Q and Tao E: Rifampicin inhibits microglial inflammation and improves neuron survival against inflammation. Brain Res (In press).

8. Li Y, Liu L, Barger SW, Mrak RE and Griffin WS: Vitamin E suppression of microglial activation is neuroprotective. J Neurosci Res 66: 163-170, 2001.

9. Jochmann R, Thurau M, Jung S, et al: O-linked N-acetylglucosaminylation of Sp1 inhibits the human immunodeficiency virus type 1 promoter. J Virol 83: 3704-3718, 2009.

10. Haltiwanger RS, Grove K and Philipsberg GA: Modulation of O-linked $\mathrm{N}$-acetylglucosamine levels on nuclear and cytoplasmic proteins in vivo using the peptide O-GlcNAcbeta-N-acetylglucosaminidase inhibitor O-(2-acetamido-2 deoxy-D-glucopyranosylidene)amino-N-phenylcarbamate. J Biol Chem 273: 3611-3617, 1998.

11. Wells L, Vosseller K and Hart GW: Glycosylation of nucleocytoplasmic proteins: signal transduction and O-GlcNAc. Science 291: 2376-2378, 2001

12. Ozcan S, Andrali SS and Cantrell JE: Modulation of transcription factor function by O-GlcNAc modification. Biochim Biophys Acta 1799: 353-364, 2010.

13. Cheung WD and Hart GW: AMP-activated protein kinase and p38 MAPK activate O-GlcNAcylation of neuronal proteins during glucose deprivation. J Biol Chem 283: 13009-13020, 2008.

14. Hart GW, Housley MP and Slawson C: Cycling of O-linked beta-N-acetylglucosamine on nucleocytoplasmic proteins. Nature 446: 1017-1022, 2007.

15. Chatham JC, Not LG, Fulop N and Marchase RB: Hexosamine biosynthesis and protein O-glycosylation: the first line of defense against stress, ischemia, and trauma. Shock 29: 431-440, 2008.
16. Yang $\mathrm{X}, \mathrm{Hu} \mathrm{W}$, Zhang Q, Wang $\mathrm{Y}$ and Sun L: Puerarin inhibits C-reactive protein expression via suppression of nuclear factor kappaB activation in lipopolysaccharide-induced peripheral blood mononuclear cells of patients with stable angina pectoris Basic Clin Pharmacol Toxicol 107: 637-642, 2010.

17. $\mathrm{Xu} \mathrm{X}$ and Zheng $\mathrm{X}$ : Potential involvement of calcium and nitric oxide in protective effects of puerarin on oxygen-glucose deprivation in cultured hippocampal neurons. J Ethnopharmacol 113: 421-426, 2007.

18. Wu CF, Bi XL, Yang JY, et al: Differential effects of ginsenosides on NO and TNF-alpha production by LPS-activated N9 microglia. Int Immunopharmacol 7: 313-320, 2007.

19. Dimayuga FO, Wang C, Clark JM, Dimayuga ER, Dimayuga VM and Bruce-Keller AJ: SOD1 overexpression alters ROS production and reduces neurotoxic inflammatory signaling in microglial cells. J Neuroimmunol 182: 89-99, 2007.

20. Bonneh-Barkay D, Reaney SH, Langston WJ and Di Monte DA: Redox cycling of the herbicide paraquat in microglial cultures. Brain Res Mol Brain Res 134: 52-56, 2005.

21 Park HY, Han MH, Park C, et al: Anti-inflammatory effects of fucoidan through inhibition of NF-kappaB, MAPK and Akt activation in lipopolysaccharide-induced BV2 microglia cells. Food Chem Toxicol (In press).

22. Tzeng SF and Huang HY: Downregulation of inducible nitric oxide synthetase by neurotrophin-3 in microglia. J Cell Biochem 90: 227-233, 2003.

23. Kim MK, Chung SW, Kim DH, et al: Modulation of age-related NF-kappaB activation by dietary zingerone via MAPK pathway. Exp Gerontol 45: 419-426, 2010.

24. Zhao S, Zhang L, Lian G, et al: Sildenafil attenuates LPS-induced pro-inflammatory responses through down-regulation of intracellular ROS-related MAPK/NF-kappaB signaling pathways in N9 microglia. Int Immunopharmacol 11: 468-474, 2011.

25. Wen J, Ribeiro R and Zhang Y: Specific PKC isoforms regulate LPS-stimulated iNOS induction in murine microglial cells. J Neuroinflammation 8: 38, 2011.

26. Liu HT, Du YG, He JL, et al: Tetramethylpyrazine inhibits production of nitric oxide and inducible nitric oxide synthase in lipopolysaccharide-induced N9 microglial cells through blockade of MAPK and PI3K/Akt signaling pathways, and suppression of intracellular reactive oxygen species. J Ethnopharmacol 129 : 335-343, 2010.

27. Rotty JD, Hart GW and Coulombe PA: Stressing the role of O-GlcNAc: linking cell survival to keratin modification. Nat Cell Biol 12: 847-849, 2010.

28. Wang Z, Udeshi ND, Slawson C, et al: Extensive crosstalk between O-GlcNAcylation and phosphorylation regulates cytokinesis. Sci Signal 3: ra2, 2010.

29. Lazarus BD, Love DC and Hanover JA: O-GlcNAc cycling: implications for neurodegenerative disorders. Int J Biochem Cell Biol 41: 2134-2146, 2009.

30. Kneass ZT and Marchase RB: Protein O-GlcNAc modulates motility-associated signaling intermediates in neutrophils. J Biol Chem 280: 14579-14585, 2005.

31. Hou Y, Wu CF, Yang JY, He X, Bi XL, Yu L and Guo T: Effects of clozapine, olanzapine and haloperidol on nitric oxide production by lipopolysaccharide-activated N9 cells. Prog Neuropsychopharmacol Biol Psychiatry 30: 1523-1528, 2006.

32. Bruce-Keller AJ, Keeling JL, Keller JN, Huang FF, Camondola S and Mattson MP: Antiinflammatory effects of estrogen on microglial activation. Endocrinology 141: 3646-3656, 2000.

33. Mayo L and Stein R: Characterization of LPS and interferongamma triggered activation-induced cell death in N9 and primary microglial cells: induction of the mitochondrial gateway by nitric oxide. Cell Death Differ 14: 183-186, 2007.

34. Chung ES, Chung YC, Bok E, et al: Fluoxetine prevents LPS-induced degeneration of nigral dopaminergic neurons by inhibiting microglia-mediated oxidative stress. Brain Res 1363: $143-150,2010$.

35. Hao Y, Yang X, Chen C, Yuan Wang, Wang X, Li M and Yu Z: STAT3 signalling pathway is involved in the activation of microglia induced by $2.45 \mathrm{GHz}$ electromagnetic fields. Int J Radiat Biol 86: 27-36, 2010.

36. Chang Y, Hsieh CY, Peng ZA, et al: Neuroprotective mechanisms of puerarin in middle cerebral artery occlusion-induced brain infarction in rats. J Biomed Sci 16: 9, 2009.

37. Platten M, Wick W, Wischhusen J and Weller M: N-[3,4dimethoxycinnamoyl]-anthranilic acid (tranilast) suppresses microglial inducible nitric oxide synthase (iNOS) expression and activity induced by interferon-gamma (IFN-gamma). Br J Pharmacol 134: 1279-1284, 2001. 
38. Weinstein JR, Swarts S, Bishop C, Hanisch UK and Moller T: Lipopolysaccharide is a frequent and significant contaminant in microglia-activating factors. Glia 56: 16-26, 2008.

39. Zhu ZT, Li HQ, Lu Y, Lou JS and Yao Z: Inhibitive effect of puerarin on increased NO production by neonatal cardiomyocytes during hypoxia/reoxygenation injury. Zhongguo Zhong Yao Za Zhi 26: 856-859, 2001 (In Chinese).

40. Yang WH, Park SY, Nam HW, et al: NFkappaB activation is associated with its O-GlcNAcylation state under hyperglycemic conditions. Proc Natl Acad Sci USA 105: 17345-17350, 2008
41. Hwang SY, Shin JH, Hwang JS, et al: Glucosamine exerts a neuroprotective effect via suppression of inflammation in rat brain ischemia/reperfusion injury. Glia 58: 1881-1892, 2010.

42. Ju Y, Hua J, Sakamoto K, Sakamoto K, Ogawa H and Nagaoka I: Modulation of TNF-alpha-induced endothelial cell activation by glucosamine, a naturally occurring amino monosaccharide. Int J Mol Med 22: 809-815, 2008. 